\title{
Enhanced carrier scattering rates in dilute magnetic semiconductors with correlated impurities
}

\author{
F. V. Kyrychenko and C. A. Ullrich \\ Department of Physics and Astronomy, University of Missouri, Columbia, Missouri 65211, USA \\ (Received 6 July 2006; revised manuscript received 22 October 2006; published 17 January 2007)
}

\begin{abstract}
In III-V dilute magnetic semiconductors such as $\mathrm{Ga}_{1-x} \mathrm{Mn}_{x} \mathrm{As}$, the impurity positions tend to be correlated, which can drastically affect the electronic transport properties of these materials. Within the memory function formalism we have derived a general expression for the current relaxation kernel in spin and charge disordered media and have calculated spin and charge scattering rates in the weak-disorder limit. Using a simple model for impurity clustering, we find a significant enhancement of the charge scattering. The enhancement is sensitive to cluster parameters and may be controllable through postgrowth annealing.
\end{abstract}

DOI: 10.1103/PhysRevB.75.045205

PACS number(s): 72.80.Ey, 78.30.Ly

\section{INTRODUCTION}

The perspective of utilizing charge and spin of the electrons for new electronic device applications has generated tremendous interest in the field of spintronics. ${ }^{1}$ A unique combination of magnetic and semiconducting properties makes dilute magnetic semiconductors (DMSs) very attractive for various spintronics applications. ${ }^{2}$ Among the family of DMSs, much attention has been paid to $\mathrm{Ga}_{1-x} \mathrm{Mn}_{x} \mathrm{As}$ since the discovery of its relatively high ferromagnetic transition temperature, ${ }^{2}$ with a current record of $T_{c}=159 \mathrm{~K}{ }^{3}$

In $\mathrm{Ga}_{1-x} \mathrm{Mn}_{x} \mathrm{As}$, unlike in II-VI DMSs, the magnetic ions in substitutional positions act as acceptors delivering one hole per ion. All $\mathrm{Ga}_{1-x} \mathrm{Mn}_{x}$ As samples are, however, heavily compensated, with hole concentrations much less than $x$. This signals the presence of substantial amounts of donor defects like arsenic antisites $\mathrm{As}_{\mathrm{Ga}}$ or interstitial manganese ions $\mathrm{Mn}_{\mathrm{I}}$ generated during low temperature molecular beam epitaxial (MBE) growth. ${ }^{4}$ The magnetic and transport properties of $\mathrm{Ga}_{1-x} \mathrm{Mn}_{x} \mathrm{As}$ depend not only on the manganese fraction $x$ but are extremely sensitive to detailed growth conditions, ${ }^{5}$ as well as to temperature and speed of postgrowth annealing. ${ }^{6-8}$ This sensitivity points to the crucial role played by the defects and their configuration, and has stimulated intense research on the structure of defects and their influence on the magnetic and transport properties of DMSs. ${ }^{9,10}$

Extended x-ray absorption fine structure measurements of low temperature MBE grown samples demonstrated the substitutional nature of $\mathrm{Mn}$ ions on the group III sublattices. ${ }^{11,12}$ As for the configuration of these defects, most theoretical models for transport in DMSs assume their random distribution. However, it was shown both experimentally ${ }^{13}$ and theoretically ${ }^{14}$ that in samples with high Curie temperature the positions of substitutional magnetic ions are correlated. These correlations might originate from the interaction between magnetic impurities, ${ }^{15}$ and, in systems with strong compensation, be due to the presence of both positively and negatively charged defects. In the latter case, Timm et al. ${ }^{9}$ found in the limit of thermal equilibrium that, driven by Coulomb attraction, the defects tend to form clusters. The main effect of such a clustering is ionic screening of the disorder Coulomb potential, which has been shown to be necessary to correctly reproduce the band gap, metal-insulator transition and shape of the magnetization curve. ${ }^{9}$
In this paper, we demonstrate that the correlation of defect positions can have a dramatic effect on electronic transport in DMSs: The conductivity of $\mathrm{Ga}_{1-x} \mathrm{Mn}_{x} \mathrm{As}$ is strongly modified through a momentum dependent impurity structure factor. We will show that the clustering ${ }^{16}$ significantly increases the charge scattering relaxation rate. At the same time, positional correlation taken alone is not sufficient to affect spin scattering: Orientational correlation of spin scatterers is also necessary.

This paper is organized as follows. In Sec. II we briefly discuss a general theory of electronic transport in spin and charge disordered media and its application to study the positional correlations of scattering centers. To highlight the effect of the impurity structure factor we then consider in Sec. III systems with one type of defect: Magnetic impurities in cation substitutional positions. Positional correlations in this case might be due to magnetic interactions. ${ }^{15}$ At the same time, in heavily compensated systems like $\mathrm{Ga}_{1-x} \mathrm{Mn}_{x} \mathrm{As}$ the defect correlations are driven by Coulomb interaction between donor and acceptor centers and are accompanied by the ionic screening of disorder potential. ${ }^{9}$ We address this situation in Sec. IV, where we consider the combined effect of ionic screening and impurity structure factor. Our conclusions are given in Sec. V.

\section{GENERAL THEORY}

We are considering band carriers moving in a system with charge and spin disorder described by the Hamiltonian

$$
\hat{H}_{I}=V^{2} \sum_{\mathbf{k}} \hat{\overrightarrow{\mathcal{U}}}(\mathbf{k}) \cdot \hat{\vec{\rho}}(-\mathbf{k}),
$$

where the four-component disorder scattering potential

$$
\hat{\overrightarrow{\mathcal{U}}}(\mathbf{k})=\frac{1}{V} \sum_{j}\left(\begin{array}{c}
U_{j}(\mathbf{k}) \\
\frac{J}{2} \hat{S}_{j}^{-} \\
\frac{J}{2} \hat{S}_{j}^{+} \\
\frac{J}{2} \hat{S}_{j}^{z}
\end{array}\right) e^{i \mathbf{k} \cdot \mathbf{R}_{j}}
$$

is coupled to the four-component charge and spin density operator of the band carriers, 


$$
\hat{\rho}^{\mu}(\mathbf{k})=\frac{1}{V} \sum_{\mathbf{q}} \sum_{\tau \tau^{\prime}}\left(\sigma^{\mu}\right)_{\tau \tau^{\prime}} \hat{a}_{\mathbf{q}-\mathbf{k}, \tau}^{+} \hat{a}_{\mathbf{q}, \tau^{\prime}}
$$

Here $\sigma^{\mu}(\mu=1,+,-, z)$ is defined via the Pauli matrices, where $\sigma^{1}$ is the $2 \times 2$ unit matrix, $\sigma^{ \pm}=\left(\sigma^{x} \pm i \sigma^{y}\right) / 2$, and the summation in Eq. (2) is performed over all defects. Localized spins in our model are treated as quantum mechanical operators coupled to the band carriers via a contact Heisenberg interaction resulting in a momentum-independent exchange constant $J$.

To describe the transport properties of DMSs we have employed the memory function formalism. ${ }^{17-19}$ The central point of this approach is the calculation of the current relaxation kernel (or memory function), whose imaginary part can be associated with the Drude relaxation rate. Our derivation of the memory function in spin- and charge-disordered media is based on an equation of motion approach for the currentcurrent response function ${ }^{20,21}$ (technical details will be published elsewhere). Here, we are particularly interested in a paramagnetic system in the long-wavelength limit, the case relevant for studying the conductivity in DMSs above $T_{c}$. In this case the memory function is obtained as

$$
\begin{aligned}
M(\omega)= & \frac{V^{2}}{n m \omega} \sum_{\mathbf{k}} k_{\alpha} k_{\beta}\left\langle\hat{\mathcal{U}}_{\mu}(-\mathbf{k}) \hat{\mathcal{U}}_{\nu}(\mathbf{k})\right\rangle_{H_{m}} \\
& \times\left[\chi_{\rho^{\mu} \rho^{\nu}}(\mathbf{k}, \omega)-\chi_{\rho^{\mu} \rho^{\nu}}^{c}(\mathbf{k}, 0)\right],
\end{aligned}
$$

where $n$ is the carrier concentration and $\chi_{\rho^{\mu}} \rho^{\nu}(\mathbf{k}, \omega)$ are charge- and spin-density response functions associated with the operators (3). The superscript $c$ in Eq. (4) refers to a clean (defect-free) system.

Equation (4) contains the set of charge- and spin-density response functions of the disordered system and, strictly speaking, should be calculated by iteration. This approach was realized in Ref. 22 to study a spin-independent system close to the metal-insulator threshold. In our case, however, we assume that the disorder is weak enough so we can approximate Eq. (4) by expanding to second order in the disorder potential $\hat{\mathcal{U}}(\mathbf{k})$, and thus replace $\chi_{\rho^{\mu} \rho^{\nu}}(\mathbf{k}, \omega)$ by its clean system counterpart $\chi_{\rho^{\mu} \rho^{\nu}}^{c}(\mathbf{k}, \omega)$.

An accurate description of carrier-mediated ferromagnetism ${ }^{23}$ and optical response ${ }^{24}$ of $\mathrm{Ga}_{1-x} \mathrm{Mn}_{x} \mathrm{As}$ would require taking the true multiband structure of the material into account. In this paper, however, we concentrate primarily on effects arising from the disorder configuration, putting less emphasis on the details of the band structure. In the evaluation of the response functions $\chi_{\rho^{\mu} \rho^{\nu}}^{c}(\mathbf{k}, \omega)$ we thus work with a simple parabolic band.

The presence of impurities, including their correlations, enters in Eq. (4) through the expression $\left\langle\hat{\mathcal{U}}_{\mu}(-\mathbf{k}) \hat{\mathcal{U}}_{\nu}(\mathbf{k})\right\rangle_{H_{m}}$. The angular brackets indicate a thermodynamical average with respect to a magnetic subsystem Hamiltonian $\hat{H}_{m}$. We assume $\hat{H}_{m}$ to be a sum of individual spin contributions corresponding to uncorrelated and noninteracting localized spins. For simplicity we neglect the time evolution of local- ized spin operators, thus assuming that the carriers move through an ensemble of frozen spins.

For the charge component $U(\mathbf{k})$ of the disorder potential operator (2) we take a Coulomb potential screened with the host material dielectric constant. Screening by the electron liquid is absorbed in the band-carrier response functions. In principle, all dynamical many-body effects contained in $\chi_{\rho^{\mu} \rho^{\nu}}$ can be treated exactly using time-dependent densityfunctional theory. ${ }^{25}$ For the purposes of the present paper, we limit ourselves to the static random phase approximation (RPA) to account for electron-electron interaction and express the density-density response function of the interacting system as

$$
\chi_{\rho^{1} \rho^{1}}(\mathbf{q}, \omega)=\frac{\chi_{0}(\mathbf{q}, \omega)}{\varepsilon_{\mathrm{RPA}}(\mathbf{q}, 0)},
$$

where $\chi_{0}(\mathbf{q}, \omega)$ is the noninteracting density-density response function of the clean system, i.e., the Lindhard function, and $\varepsilon_{\text {RPA }}(\mathbf{q}, 0)$ is the static RPA dielectric function. ${ }^{26}$ The spin response functions in the paramagnetic state are not affected by electron-electron interactions on the RPA level, and they can also be expressed in terms of the Lindhard function $\chi_{0}(\mathbf{q}, \omega)$.

\section{SUBSTITUTIONAL MAGNETIC IONS}

In order to separate the effect of the impurity structure factor from other effects of clustering like ionic screening we first consider a system with only one type of defect: $\mathrm{Mn}^{2+}$ ions in cation substitutional positions. All defects in Eq. (2) thus carry localized spin and produce the same charge potential.

Let us consider the correlated product of two components of the disorder potential in Eq. (4). First, we separate the same-ion $\left(j=j^{\prime}\right)$ and pair $\left(j \neq j^{\prime}\right)$ contributions:

$$
\begin{aligned}
\left\langle\hat{\mathcal{U}}_{\mu}(-\mathbf{k}) \hat{\mathcal{U}}_{\nu}(\mathbf{k})\right\rangle_{H_{m}}= & \frac{n_{i}}{V}\left\langle\hat{U}_{\mu}(-\mathbf{k}) \hat{U}_{\nu}(\mathbf{k})\right\rangle_{H_{m}} \\
& +\frac{1}{V^{2}}\left\langle\hat{U}_{\mu}(-\mathbf{k})\right\rangle_{H_{m}}\left\langle\hat{U}_{\nu}(\mathbf{k})\right\rangle_{H_{m}} \\
& \times \sum_{j \neq j^{\prime}} e^{i \mathbf{k} \cdot\left(\mathbf{R}_{j^{\prime}}-\mathbf{R}_{j}\right)},
\end{aligned}
$$

where $n_{i}$ is the impurity concentration, and in the second term the average of the product becomes a product of averages for noninteracting spins. Random impurities are taken into account through the first term on the right-hand side of Eq. (6), while the second term contributes only if there are correlations in the impurity positions. For spin scattering, however, spatial correlations are not sufficient for the pair term to survive. Indeed, if any of the indices $\mu, \nu$ correspond to a spin component (e.g., $\mu=z$ ), then the second term in Eq. (6) is proportional to the average spin and vanishes if $\left\langle\hat{S}_{z}\right\rangle$ $=0$, regardless of spatial correlations. The presence of two sources of randomness is a characteristic feature of spin scattering. In terms of scattering, localized spins are correlated if they exhibit both positional and orientational correlations. 
Note that the presence of a macroscopic magnetization is not necessary for spins to be correlated. What counts in the scattering is the short-range orientational correlation that might be present even in a macroscopically paramagnetic system.

For the charge-scattering term $(\mu, \nu=1)$, we have

$$
\left\langle\hat{\mathcal{U}}_{1}(-\mathbf{k}) \hat{\mathcal{U}}_{1}(\mathbf{k})\right\rangle_{H_{m}}=\left|U_{1}(\mathbf{k})\right|^{2} \frac{n_{i}}{V} S(\mathbf{k}),
$$

with the structure factor

$$
S(\mathbf{k})=1+\frac{\Omega_{0}}{V x} \sum_{j \neq j^{\prime}} e^{i \mathbf{k} \cdot\left(\mathbf{R}_{j^{\prime}}-\mathbf{R}_{j}\right)},
$$

where $x$ is the molar fraction of magnetic ions in the sample and $\Omega_{0}$ is the elementary cell volume. Let us introduce a pair distribution function $P(\mathbf{R})$, normalized as

$$
\frac{1}{V} \int_{V} P(\mathbf{R}) d \mathbf{R}=x,
$$

which describes the probability of finding another magnetic ion at a distance $\mathbf{R}$ from a given ion. With the help of $P(\mathbf{R})$, we approximate Eq. (8) as

$$
S(\mathbf{k}) \approx 1+\frac{1}{\Omega_{0}} \int_{V} P(\mathbf{R}) \cos (\mathbf{k} \cdot \mathbf{R}) d \mathbf{R} .
$$

For a random impurity distribution one has $P(\mathbf{R})=x$, and the second term in Eq. (10) vanishes. The structure factor $S(\mathbf{k})$ is then equal to 1 , which implies a contribution only of the same-ion term in Eq. (6).

To study the effect of correlations in the defect positions (higher probability to find magnetic impurities close to each other), we employ a simple model expression for a pair distribution function $P(\mathbf{R})$, assuming it to be a piecewise constant, spherically symmetrical function of the form

$$
P(R)= \begin{cases}x_{c}, & R<R_{c}, \\ x_{d}, & R_{c}<R<R_{d}, \\ x, & R>R_{d} .\end{cases}
$$

The first region corresponds to a cluster of radius $R_{c}$ with effective impurity concentration $x_{c}>x$; the second region is a depletion layer with $x_{c}<x$, necessary to preserve the average impurity concentration in the sample. The width of the depletion layer is determined by the normalization condition (9). The impurity concentration within the depletion layer has a minor impact on the final results, and to keep things simple we fix it to $x_{d}=x / 2$.

The remaining two parameters, $R_{c}$ and $x_{c}$, describe the cluster structure and are in general independent. We can relate them, however, if we fix the average number $N$ of the impurity ions within the cluster:

$$
\frac{4 \pi R_{c}^{3}}{3 \Omega_{0}} x_{c}=N .
$$

This seems reasonable for modeling the effect of annealing on low-temperature grown DMS samples, where one may assume that the total number of $\mathrm{Mn}^{2+}$ substitutional ions

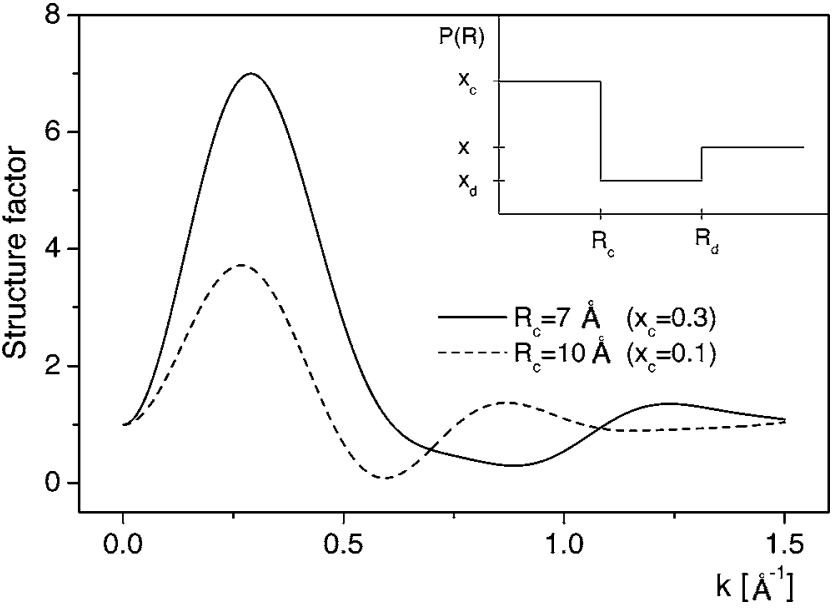

FIG. 1. Momentum-dependent impurity structure factor (13) for different values of cluster radius $R_{c}$. Number of ions within the cluster $N=10$; average impurity concentration in the sample $x$ $=0.05$. Inset: Schematic plot of the pair distribution function (11).

within the cluster is conserved while the cluster size (and thus density) may vary. In the following we will use $N=10$, consistent with the results of Monte Carlo simulations. ${ }^{9}$

Our model pair correlation function (11) yields the following momentum-dependent impurity structure factor:

$$
\begin{aligned}
S(k)= & 1+\frac{2 \pi x}{k^{3} \Omega_{0}}\left\{\frac{2 x_{c}-x}{x}\left[\sin \left(k R_{c}\right)-k R_{c} \cos \left(k R_{c}\right)\right]\right. \\
& \left.-\left[\sin \left(k R_{d}\right)-k R_{d} \cos \left(k R_{d}\right)\right]\right\} .
\end{aligned}
$$

Figure 1 shows $S(k)$ for two different values of the cluster radius $R_{c}$, which in principle can be controlled by annealing. As expected, the structure factor oscillates with decreasing amplitude, with a larger first maximum for smaller cluster size.

Having an expression for the correlated product of the components of disorder potential, Eq. (4) can be directly evaluated and the memory function becomes the sum of charge and spin contributions

$$
M(\omega)=\frac{1}{\tau_{n}}+\frac{1}{\tau_{s}},
$$

with

$$
\frac{1}{\tau_{n}(\omega)}=A \int_{0}^{\infty} k^{4} S(k) \frac{\left|U_{1}(k)\right|^{2}}{\varepsilon_{\mathrm{RPA}}(k)} \frac{\chi_{0}(k, \omega)-\chi_{0}(k)}{\omega} d k
$$

and

$$
\frac{1}{\tau_{s}(\omega)}=A \frac{J^{2}}{4} S_{\mathrm{Mn}}\left(S_{\mathrm{Mn}}+1\right) \int_{0}^{\infty} k^{4} \frac{\chi_{0}(k, \omega)-\chi_{0}(k)}{\omega} d k .
$$

Here $S_{\mathrm{Mn}}=5 / 2$ is the localized spin of magnetic impurities, and the common prefactor is given by $A=\left(n_{i} / n\right) V^{2} / 6 \pi^{2} m$. The imaginary parts of Eqs. (15) and (16) represent the energy dependent charge- and spin-scattering contributions to 


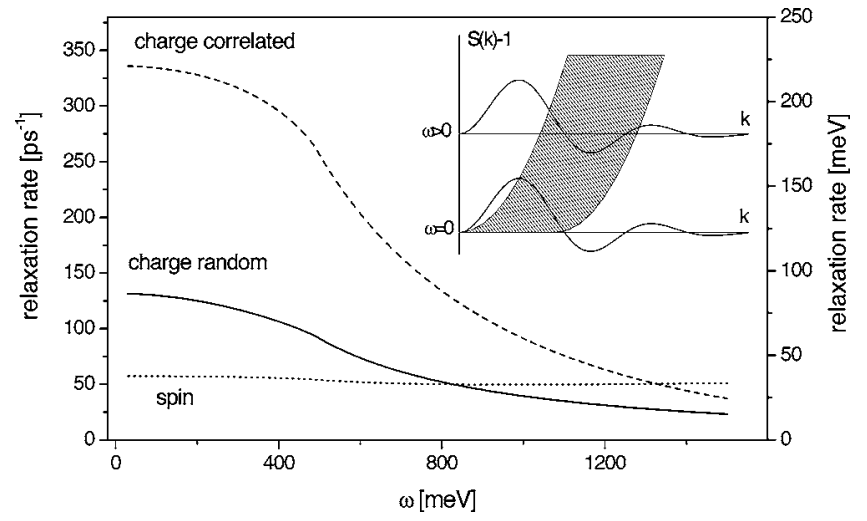

FIG. 2. Frequency dependence of charge (15) and spin (16) scattering relaxation rates, for impurity clusters with $R_{c}=10 \AA, x_{c}$ $=0.1$, and hole concentration $p=0.5$ hole per magnetic ion. Inset: Illustration of the frequency dependence of the cluster enhancement; see text.

the Drude relaxation rate. Generally speaking, within our model the relaxation times $\tau_{n(s)}(\omega, \mathbf{q})$ are also momentum dependent. In the present paper, however, we consider only the long-wavelength limit, setting $\mathbf{q} \rightarrow 0$. Note that the momentum-dependent impurity structure factor $S(k)$ does not appear in the spin term (16).

Throughout this paper we performed our calculations for the case of $\mathrm{Ga}_{0.95} \mathrm{Mn}_{0.05} \mathrm{As}$. Material parameters used are heavy hole effective mass $m=0.5 m_{0}$, dielectric constant $\varepsilon$ $=13$, and exchange constant $V J=55 \mathrm{meV} \mathrm{nm}^{3}$, which corresponds to the widely used DMS $p$ - $d$ exchange constant $N_{0} \beta=1.2 \mathrm{eV} .{ }^{23}$

In Fig. 2 we plot the imaginary parts of Eqs. (15) and (16) as a function of $\omega$. Both spin and charge relaxation rates demonstrate the frequency dependencies that one might expect for momentum-independent scattering and for Coulomb scattering. In agreement with earlier golden rule estimates, ${ }^{27}$ the charge scattering dominates the spin scattering in the dc limit. However, the latter contribution is clearly not negligible, and reaches the same order of magnitude as charge scattering for higher frequencies. Both contributions should therefore be taken into account simultaneously.

Correlation in impurity positions results in a significant increase of the charge scattering contribution at low frequency, while for higher $\omega$ this enhancement decreases. The origin of this effect is illustrated in the inset in Fig. 2. The presence of impurity clusters selects excitations within a finite momentum window defined by the impurity structure factor. On the other hand, the region of one-particle excitations is given by the imaginary part of the Lindhard function $\chi_{0}$ (shaded region in Fig. 2). The maximum enhancement of the relaxation rate corresponds to maximum overlap of the selection window with the one-particle spectrum, which happens at low frequency. For higher $\omega$ the window falls outside of the available excitation spectrum, reducing the relaxation rate enhancement.

\section{STRONGLY COMPENSATED SYSTEMS}

By considering only one type of defect (magnetic ions in cation substitutional positions) we have discussed in the pre- vious section the impact of the impurity structure factor on the electron transport and showed that it results in an increase of the carrier relaxation rate. However, the appearance of the structure factor is not the only effect of the impurity positional correlations in DMS materials. It was shown ${ }^{9}$ that in strongly compensated systems, like $\mathrm{Ga}_{1-x} \mathrm{Mn}_{x} \mathrm{As}$, the presence of defects of both charges results in their mutual positional correlations and thus reduces significantly the charge disorder potential. This effect of ionic screening should obviously lead to a decrease of carrier relaxation rates. A meaningful analysis of the consequences of the positional correlation of impurities on transport properties of DMSs should therefore take into account both contributions. In this section we consider systems with different types of defects and study the combined effect of the impurity structure factor and ionic screening of the disorder potential on the transport properties of $\mathrm{Ga}_{1-x} \mathrm{Mn}_{x}$ As.

In this case, the disorder Hamiltonian (1) is generalized to

$$
\hat{H}_{I}=V^{2} \sum_{\mathbf{k}}\left[\hat{\overrightarrow{\mathcal{U}}}^{1}(\mathbf{k})+\hat{\overrightarrow{\mathcal{U}}}^{2}(\mathbf{k})+\cdots\right] \cdot \hat{\vec{\rho}}(-\mathbf{k}),
$$

where $\hat{\mathcal{U}}^{i}(\mathbf{q})$ describe different types of defects $\left(\mathrm{Mn}_{\mathrm{Ga}}, \mathrm{Mn}_{\mathrm{I}}\right.$, $\mathrm{As}_{\mathrm{Ga}}$, etc.). Potentials are, in general, four component quantities, but for pure charge or pure spin defects some components may vanish. For simplicity we will take into account only two types of defects: $\mathrm{Mn}_{\mathrm{Ga}}$, which we considered before, and $\mathrm{Mn}_{\mathrm{I}}$, manganese ions in the interstitial positions, which we treat for our purposes as spinless double donors. The number of $\mathrm{Mn}_{\mathrm{I}}$ double donors is determined by the number of holes and $\mathrm{Mn}_{\mathrm{Ga}}$ acceptors through the charge neutrality relation.

The product of the components of the disorder potential $\left\langle\hat{\mathcal{U}}_{\mu}^{i}(\mathbf{k}) \hat{\mathcal{U}}_{\nu}^{i^{\prime}}(-\mathbf{k})\right\rangle_{H_{m}}$ now contains both diagonal $\left(i=i^{\prime}\right)$ and nondiagonal $\left(i \neq i^{\prime}\right)$ terms. Diagonal products have the same form (6) as in the case of a single type of defect, while the off-diagonal terms involve essentially different defects and thus contain only the pair term. The off-diagonal cross product of the charge components $(\mu=\nu=1)$ of donor and acceptor potentials

$$
\left\langle\hat{\mathcal{U}}_{1}^{i}(\mathbf{k}) \hat{\mathcal{U}}_{1}^{i^{\prime}}(-\mathbf{k})\right\rangle_{H_{m}}=\frac{1}{V^{2}} U_{n}^{i}(\mathbf{k}) U_{n}^{i^{\prime}}(-\mathbf{k}) \sum_{j \neq j^{\prime}} e^{i \mathbf{k} \cdot\left(\mathbf{R}_{j^{\prime}}^{i^{\prime}}-\mathbf{R}_{j}^{i}\right)}
$$

describes in our model the effect of ionic screening.

The general expression for products of the charge components of the disorder potential (including both diagonal and off-diagonal terms) can be written as

$$
\left\langle\hat{\mathcal{U}}_{n}^{i}(\mathbf{k}) \hat{\mathcal{U}}_{n}^{i^{\prime}}(-\mathbf{k})\right\rangle_{H_{m}}=U_{n}^{i}(\mathbf{k}) U_{n}^{i^{\prime}}(-\mathbf{k}) \frac{n_{i}}{V} S_{i i^{\prime}}(\mathbf{k}),
$$

with the structure factor

$$
S_{i i^{\prime}}(\mathbf{k})=\delta_{i i^{\prime}}+\frac{n_{i^{\prime}} V}{\Omega_{0}} \int_{V} P_{i i^{\prime}}(R) \cos (\mathbf{k} \cdot \mathbf{R}) d \mathbf{R} .
$$

Note that cross product terms vanish if there is no correlation in acceptor and donor positions. 


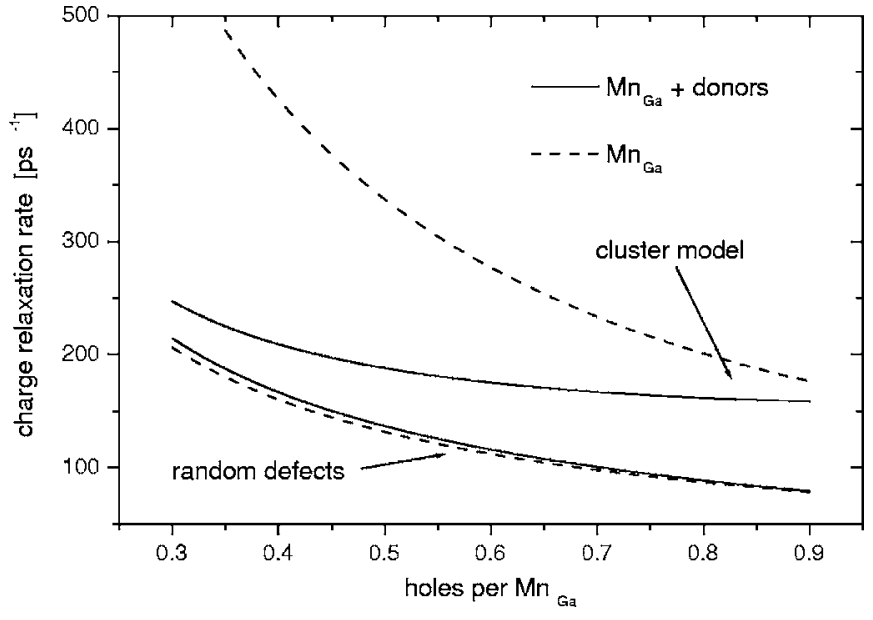

FIG. 3. Charge contribution to the relaxation rate as a function of the degree of compensation (number of holes per substitutional Mn ion). Solid lines include effects of ionic screening. The cluster model assumed $R_{c}=10 \AA$ and $x_{c}=0.1$.

In the case where our system is characterized by two types of defects we need three pair distribution functions $P_{i i^{\prime}}$. In our calculations we take them in the form (11) with the same values of cluster parameters $R_{c}$ and $R_{d}$. In addition, we assume that $\mathrm{Mn}_{\mathrm{I}}$ double donors always sit near one of the acceptors, forming a substitutional-interstitial pair.

In Fig. 3 we plot the charge contribution to the carrier relaxation rate (15) as a function of the degree of compensation (number of holes per substitutional magnetic ion). The dashed lines neglect the presence of donors, while the solid lines take into account the effects of ionic screening. It is seen that for random defect distribution the effect of ionic screening is negligible while in our cluster model it substantially reduces the charge scattering. Nevertheless, this reduction is smaller than the enhancement of relaxation rate due to impurity structure factor. The net effect of clustering is an increase of scattering rate for any degree of compensation. This increase, however, is more pronounced in systems with lower compensation (higher number of holes and lower number of donors per $\mathrm{Mn}_{\mathrm{Ga}}$ ) where the ionic screening is weaker.

Another effect is the interplay between ionic screening and band carrier screening of the disorder potential. Postgrowth annealing, which is widely used to increase $T_{c}$ in $\mathrm{Ga}_{1-x} \mathrm{Mn}_{x}$ As samples, removes highly mobile $\mathrm{Mn}_{\mathrm{I}}$ double donors from the system. ${ }^{8}$ From the point of view of electron scattering the consequence of this process is twofold. First, it increases the number of holes in the valence band, thus enhancing the screening of disorder by mobile carriers. On the other hand, reducing the number of donors suppresses the ionic screening of the disorder potential. As shown in Fig. 4, the competition between these two processes results in a nonmonotonic dependence of the charge relaxation rate on the degree of compensation in samples with relatively dense clusters, where ionic screening plays an important role.

Along with the removal of interstitials, postgrowth annealing also affects the relative positions of substitutional Mn ions. To get some insight into the impact of cluster configuration on the relaxation rates, we plot in Fig. 5 the cluster enhancement factor

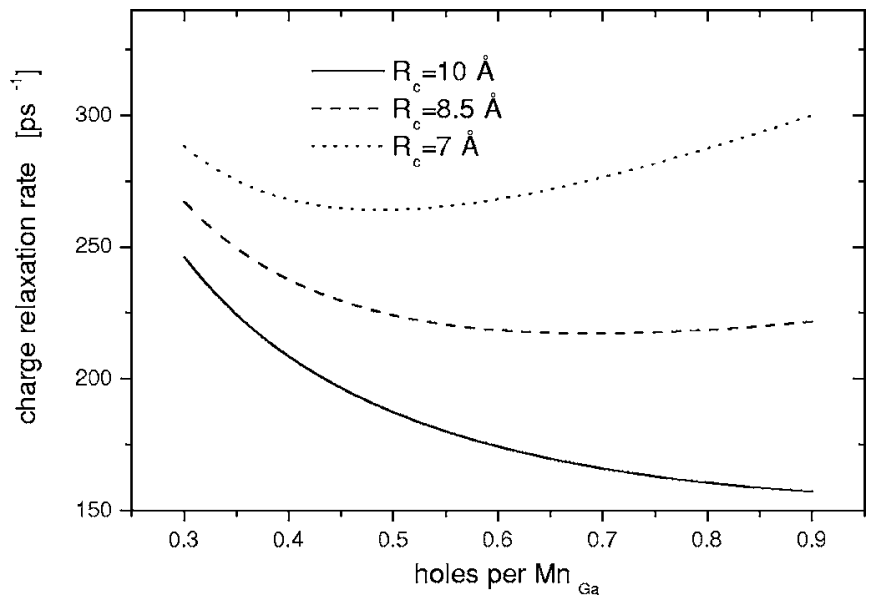

FIG. 4. Charge relaxation rate as a function of compensation for different cluster sizes (average number of $\mathrm{Mn}_{\mathrm{Ga}}$ ions within the cluster is fixed to $N=10$ ). The interplay between ionic and band carrier screening results in a nonmonotonic dependence for dense clusters.

$$
\xi=\frac{\tau_{n}^{-1}+\tau_{s}^{-1}}{\left(\tau_{n}^{R}\right)^{-1}+\left(\tau_{s}^{R}\right)^{-1}}
$$

as a function of cluster radius for different degrees of compensation. The enhancement factor is defined as the ratio of the total (charge plus spin) relaxation rates for correlated and random impurity distributions. Recall that the average number of magnetic ions within the cluster is fixed to $N=10$, and the cluster radius $R_{c}$ is related to the concentration of magnetic ions within the cluster $x_{c}$ through Eq. (12). The enhancement is strongest (over $100 \%$ ) for weakly compensated systems and is quite sensitive to cluster configuration. The latter will be sensitive to postgrowth annealing.

\section{CONCLUSIONS}

To summarize, using the memory function formalism we have considered transport in charge and spin disordered me-

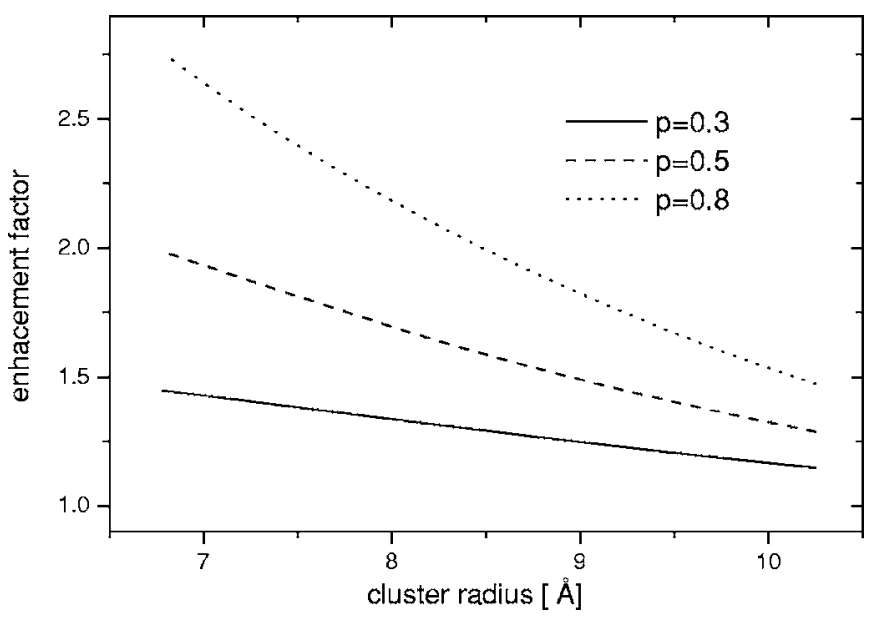

FIG. 5. Relaxation rate enhancement (21) due to correlation in impurity positions as a function of cluster size. The average number of magnetic ions within the cluster is fixed to $N=10$. 
dia with potential application for DMSs, with particular emphasis on nonrandomness of impurity positions in $\mathrm{Ga}_{1-x} \mathrm{Mn}_{x}$ As. We have shown that positional correlations of the magnetic impurities alone do not affect spin scattering: Orientational correlations are also necessary. For charge scattering, impurity clustering gives rise to a momentumdependent impurity structure factor which substantially modifies the transport properties of the material. The combined effect of impurity structure factor and ionic screening of the disorder potential typically leads to more than $50 \%$ enhancement of relaxation rates in systems with positional correlations. These results should give valuable insight into the effects of annealing on low temperature grown DMS samples.

Finally, the discussion in this paper was limited to DMS in the paramagnetic state. However, it is well known that magnetic ordering in DMS can have a dramatic influence on transport properties. ${ }^{28}$ Our approach should be well suited to study these effects.

\section{ACKNOWLEDGMENTS}

This work was supported by DOE Grant No. DE-FG02$05 \mathrm{ER} 46213$
${ }^{1}$ G. A. Prinz, Science 282, 1660 (1998).

${ }^{2}$ H. Ohno, Science 281, 951 (1998).

${ }^{3}$ K. W. Edmonds, P. Boguslawski, K. Y. Wang, R. P. Campion, S. N. Novikov, N. R. S. Farley, B. L. Gallagher, C. T. Foxon, M. Sawicki, T. Dietl, M. B. Nardelli, and J. Bernholc, Phys. Rev. Lett. 92, 037201 (2004).

${ }^{4}$ H. Munekata, H. Ohno, S. von Molnar, A. Segmuller, L. L. Chang, and L. Esaki, Phys. Rev. Lett. 63, 1849 (1989).

${ }^{5}$ H. Shimizu, T. Hayashi, T. Nishinaga, and M. Tanaka, Appl. Phys. Lett. 74, 398 (1999).

${ }^{6}$ T. Hayashi, Y. Hashimoto, S. Katsumoto, and Y. Iye, Appl. Phys. Lett. 78, 1691 (2001).

${ }^{7}$ S. J. Potashnik, K. C. Ku, S. H. Chun, J. J. Berry, N. Samarth, and P. Schiffer, Appl. Phys. Lett. 79, 1495 (2001).

${ }^{8}$ K. M. Yu, W. Walukiewicz, T. Wojtowicz, I. Kuryliszyn, X. Liu, Y. Sasaki, and J. K. Furdyna, Phys. Rev. B 65, 201303(R) (2002).

${ }^{9}$ C. Timm, F. Schäfer, and F. von Oppen, Phys. Rev. Lett. 89, 137201 (2002).

${ }^{10}$ X. Y. Cui, J. E. Medvedeva, B. Delley, A. J. Freeman, N. Newman, and C. Stampfl, Phys. Rev. Lett. 95, 256404 (2005).

${ }^{11}$ R. Shioda, K. Ando, T. Hayashi, and M. Tanaka, Phys. Rev. B 58, 1100 (1998).

${ }^{12}$ Y. L. Soo, G. Kioseoglou, S. Kim, X. Chen, H. Luo, Y. H. Kao, H.-J. Lin, H. H. Hsieh, T. Y. Hou, C. T. Chen, Y. Sasaki, X. Liu, and J. K. Furdyna, Phys. Rev. B 67, 214401 (2003).

${ }^{13}$ Y. L. Soo, S. Kim, Y. H. Kao, A. J. Blattner, B. W. Wessels, S. Khalid, C. Sanchez-Hanke, and C.-C. Kao, Appl. Phys. Lett. 84, 481 (2004).
${ }^{14}$ G. Bouzerar, T. Ziman, and J. Kudrnovsky, Appl. Phys. Lett. 85, 4941 (2004).

${ }^{15}$ M. van Schilfgaarde and O. N. Mryasov, Phys. Rev. B 63, 233205 (2001).

${ }^{16}$ By "clustering" we mean the positional correlation of Mn ions in group-III sublattice substitutional positions, and not the presence of MnAs precipitates.

${ }^{17}$ W. Götze, Philos. Mag. B 43, 219 (1981).

${ }^{18}$ D. Belitz and S. Das Sarma, Phys. Rev. B 34, 8264 (1986).

${ }^{19}$ C. A. Ullrich and G. Vignale, Phys. Rev. B 65, 245102 (2002); , 70, 239903(E) (2004).

${ }^{20}$ W. Götze and P. Wölfle, Phys. Rev. B 6, 1226 (1972).

${ }^{21}$ G. F. Giuliani and G. Vignale, Quantum Theory of the Electron Liquid (Cambridge University Press, Cambridge, UK, 2005).

${ }^{22}$ A. Gold and W. Götze, Phys. Rev. B 33, 2495 (1986).

${ }^{23}$ T. Dietl, H. Ohno, and F. Matsukura, Phys. Rev. B 63, 195205 (2001).

${ }^{24}$ J. Sinova, T. Jungwirth, S.-R. Eric Yang, J. Kučera, and A. H. MacDonald, Phys. Rev. B 66, 041202(R) (2002).

${ }^{25}$ Time-Dependent Density Functional Theory, edited by M. A. L. Marques, C. A. Ullrich, F. Nogueira, A. Rubio, K. Burke, and E. K. U. Gross, Lecture Notes in Physics Vol. 706 (Springer, Berlin, 2006).

${ }^{26}$ G. D. Mahan, Many-Particle Physics, 3rd ed. (Plenum, New York, 2000).

${ }^{27}$ T. Jungwirth, M. Abolfath, J. Sinova, J. Kucera, and A. H. MacDonald, Appl. Phys. Lett. 81, 4029 (2002).

${ }^{28}$ T. Omiya, F. Matsukara, T. Dietl, Y. Ohno, T. Sakon, M. Motokawa, and H. Ohno, Physica E (Amsterdam) 7, 976 (2000). 